\title{
Generalized implicit viscosity approximation method for multivalued mappings in CAT(0) spaces
}

https://doi.org/10.1515/dema-2019-0031

Received April 10, 2019; accepted August 4, 2019

\begin{abstract}
We prove strong convergence of the sequence generated by implicit viscosity approximation method involving a multivalued nonexpansive mapping in framework of CAT(0) space. Under certain appropriate conditions on parameters, we show that such a sequence converges strongly to a fixed point of the mapping which solves a variational inequality. We also present some convergence results for the implicit viscosity approximation method in complete $\mathbb{R}$-trees without the endpoint condition.
\end{abstract}

Keywords: viscosity approximation method, variational inequality, multivalued nonexpansive mapping, $\operatorname{CAT}(0)$ space

MSC: 47J25, 47H09, 37C25

\section{Introduction}

Let $C$ be a nonempty closed convex subset of a Hilbert space $E$ and $T: C \rightarrow C$, the fixed point set of $T$ is denoted by $F(T)$, that is, $F(T)=\{x \in C: x=T x\}$. A mapping $T: C \rightarrow C$ said to be a nonexpansive mapping if $\|T x-T y\| \leq\|x-y\|$ for all $x, y \in C$.

In the past few decades, several iterative schemes have been introduced to approximate fixed point of nonexpansive mappings.

In 2000, Moudafi [1] introduced a new iterative method for approximating the fixed point of nonexpansive mappings. An approximating sequence $\left\{x_{n}\right\}$ is generated through the following scheme:

$$
\begin{aligned}
& x_{0} \in C \text { arbitrarily chosen } \\
& x_{n+1}=\alpha_{n} f\left(x_{n}\right)+\left(1-\alpha_{n}\right) T x_{n} ; \quad n \geq 0,
\end{aligned}
$$

where $\left\{\alpha_{n}\right\}$ is a sequence in $(0,1)$ and $f: C \rightarrow C$ is a contraction mapping with contractive factor $k \in(0,1)$. This method is commonly known as viscosity approximation method.

Moudafi has shown that under certain conditions, the sequence $\left\{x_{n}\right\}$ not only converges strongly to a fixed point $x_{\star}$ of $T$ but also solves the following variational inequality:

$$
\left\langle f x_{\star}-\chi_{\star}, \chi_{\star}-x\right\rangle \geq 0 \text {, where } x \in F(T) .
$$

\footnotetext{
Mujahid Abbas: Department of Mathematics, Government College University, Lahore 54000, Pakistan; Department of Mathematics and Applied Mathematics, University of Pretoria, Pretoria 0002, South Africa; E-mail: abbas.mujahid@gmail.com Hira Iqbal: Department of Sciences and Humanities, National University of Computer and Emerging Sciences, Lahore Campus, Pakistan; E-mail: hira.iqbal@nu.edu.pk

*Corresponding Author: Manuel de la Sen: Institute of Research and Development of Processes, University of the Basque Country, Campus of Leioa (Bizkaia) - P.O. Box 644- Bilbao, Barrio Sarriena, 48940- Leioa, Spain;

E-mail: manuel.delasen@ehu.eus
} 
The viscosity approximation has attracted the attention of several mathematicians due to its applications in monotone inclusions, convex optimization, and linear programming, etc.

In 2015, Xu et al. [2] modified the viscosity method and introduced an implicit midpoint rule for nonexpansive mappings as follows:

$$
\begin{aligned}
& x_{0} \in C \quad \text { arbitrarily chosen } \\
& x_{n+1}=\alpha_{n} f\left(x_{n}\right)+\left(1-\alpha_{n}\right) T\left(\frac{x_{n}+x_{n+1}}{2}\right) ; \quad n \geq 0 .
\end{aligned}
$$

The authors showed that viscosity implicit midpoint rule converges strongly to a fixed point of $T$ and also solves the variational inequality (1).

Ke and Ma [3] studied the generalized viscosity implicit rules of nonexpansive mappings.

They introduced the following schemes and proved convergence theorems under certain assumptions imposed on the sequences of parameters $\alpha_{n}$, and $\beta_{n}$ :

$$
x_{n+1}=\alpha_{n} f\left(x_{n}\right)+\left(1-\alpha_{n}\right) T\left(\beta_{n} x_{n}+\left(1-\beta_{n}\right) x_{n+1}\right),
$$

and

$$
x_{n+1}=\alpha_{n} x_{n}+\beta_{n} f\left(x_{n}\right)+\gamma_{n} T\left(s_{n} x_{n}+\left(1-s_{n}\right) x_{n+1}\right) .
$$

Recently, many authors have generalized results on nonlinear mappings to more general spaces than Hilbert spaces such as CAT(0) spaces such as [4-6]. The study of viscosity approximation methods has been extended to various directions. Wu and Zhao [7] proved the viscosity approximation results for multivalued nonexpansive mappings in the setup of Hilbert and Banach spaces and obtained a relationship between fixed point of the such mappings and the solution of the variational inequality problem.

Preechasilp [8] extended the results in [2] to CAT(0) spaces. Panyanak and Suantai [9] extended the results in [10] for a multivalued nonexpansive mapping in the setting of CAT(0) spaces. Xiong and Lan [11] and [12] studied a two-step viscosity iteration approximation methods for approximating the fixed points of multivalued nonexpansive mappings in CAT(0) spaces.

In this paper, motivated by the work in [8] and [11, 12], the generalized implicit viscosity approximation scheme presented by Ke and Ma [3] is extended and generalized in the following ways:

(a) underlying space possess a nonlinear structure;

(b) a two step implicit viscosity scheme is introduced for a multivalued nonexpansive mapping.

Consider the following two step implicit viscosity approximation method for multivalued nonexpansive mapping in the framework of CAT(0) spaces. Let $\alpha_{n}, \beta_{n} \in(0,1)$, and $x_{0} \in C$ chosen arbitrarily. Define

$$
\begin{aligned}
x_{n+1} & =\alpha_{n} f\left(x_{n}\right) \oplus\left(1-\alpha_{n}\right) u_{n} ; \quad u_{n} \in T y_{n} ; \\
y_{n} & =\beta_{n} x_{n} \oplus\left(1-\beta_{n}\right) z_{n+1} ; \quad z_{n+1} \in T x_{n+1},
\end{aligned}
$$

such that $d\left(z_{n}, z_{n+1}\right) \leq d\left(x_{n}, x_{n+1}\right), d\left(u_{n}, u_{n+1}\right) \leq d\left(y_{n}, y_{n+1}\right)$ and $d\left(x_{n}, z_{n+1}\right) \leq d\left(x_{n}, x_{n+1}\right)$ and

$$
\begin{aligned}
x_{n+1} & =\alpha_{n} f\left(x_{n}\right) \oplus\left(1-\alpha_{n}\right) u_{n} ; \quad u_{n} \in T y_{n} ; \\
y_{n} & =\beta_{n} x_{n} \oplus\left(1-\beta_{n}\right) x_{n+1},
\end{aligned}
$$

such that $d\left(u_{n}, u_{n+1}\right) \leq d\left(y_{n}, y_{n+1}\right)$. We prove the convergence of the above implicit viscosity iterative processes to the fixed point of the multivalued nonexpansive map under some appropriate conditions on the parameters. We also show that this fixed point solves a variational inequality. Now, we recall some definitions and lemmas to be used in the sequel. A subset $C$ of a metric space $(E, d)$ is called proximal if for any $x \in E$, there exists an element $y \in C$ such that

$$
d(x, y)=d(x, C)=\inf \{d(x, z): z \in C\} .
$$


We denote by $K(C)$ and $C B(X)$, the collection of all nonempty compact subsets of $C$ and the collection of all nonempty closed bounded subsets of $C$, respectively. The Hausdorff distance induced by metric $d$ on $E$ is given by

$$
H(A, B)=\max \left\{\sup _{a \in A} d(a, B), \sup _{b \in B} d(b, A)\right\} .
$$

Let $T: C \rightarrow 2^{C}$, where $2^{C}$ is the collection of all nonempty subsets of $C$. An element $x \in C$ is called a fixed point of $T$, if $x \in T x$. The set of all fixed points of $T$ will be denoted by $F(T)$.

Let $x, y \in E$. A geodesic path joining $x$ to $y$ (or, more briefly, a geodesic from $x$ to $y$ ) is a map $c$ from a closed interval $[0, a]$ to $E$ such that $c(0)=x, c(a)=y$, and $d(c(s), c(t))=|t-s|$ for all $t, s \in[0, a]$. In particular, $c$ is an isometry and $d(x, y)=a$. The image of $c$ is called a geodesic (or metric) segment joining $x$ and $y$. When it is unique, the geodesic segment is denoted by $[x, y]$. The space $E$ is called a geodesic if every two points of $E$ are joined by a geodesic, and $E$ is said to be uniquely geodesic if there is exactly one geodesic joining $x$ and $y$ for each $x, y \in E$. A subset $C \subset X$ is said to be convex if $C$ includes every geodesic segment joining any two of its points.

A geodesic triangle $\Delta(p, q, r)$ in a geodesic space $(E, d)$ consists of three points $p, q, r$ in $E$ (called vertices of $\Delta$ ) and a choice of three geodesic segments $[p, q],[q, r],[r, p]$ ( edges of $\Delta$ ) joining them. A comparison triangle for a geodesic triangle $\Delta(p, q, r)$ in $E$ is a triangle $\bar{\Delta}(\bar{p}, \bar{q}, \bar{r})$ in the Euclidean plane $\mathbb{R}^{2}$ such that $d_{\mathbb{R}^{2}}(\bar{p}, \bar{q})=d(p, q), d_{\mathbb{R}^{2}}(\bar{q}, \bar{r})=d(q, r)$ and $d_{\mathbb{R}^{2}}(\bar{r}, \bar{p})=,d(r, p)$. A triangle $\triangle(\bar{p}, \bar{q}, \bar{r})$ having vertices $\bar{p}, \bar{q}, \bar{r} \in \mathbb{R}^{2}$ is called a comparison triangle of $\Delta(p, q, r)$ in $E$.

A point $\bar{u} \in[\bar{p}, \bar{q}]$ is said to be a comparison point for $u \in[p, q]$ if $d(p, u)=d_{\mathbb{R}^{2}}(\bar{p}, \bar{u})$. Similarly, comparison points on $[\bar{q}, \bar{r}]$ and $[\bar{r}, \bar{p}]$ can be defined.

Suppose that $\Delta$ is a geodesic triangle in $E$ and $\bar{\Delta}$ is a comparison triangle for $\Delta$. A geodesic space is said to be a CAT(0) space, if all geodesic triangles of appropriate size satisfy the following comparison axiom called CAT(0) inequality:

$$
d(u, v) \leq d_{\mathbb{R}^{2}}(\bar{u}, \bar{v}), \quad \text { for all } u, v \in \Delta, \quad \bar{u}, \bar{v} \in \bar{\Delta} .
$$

Every $\operatorname{CAT}(0)$ space is a uniquely geodesic and any complete and simply connected Riemannian manifold having nonpositive sectional curvature is a CAT(0) space. Other examples of CAT(0) spaces include preHilbert spaces, R-trees, Euclidean buildings and complex Hilbert ball with a hyperbolic metric as special case (see, for example, [13-15]).

Let $C$ be a nonempty closed convex subset of a complete CAT(0) space $(E, d)$. Then, for any $x \in E$ there exists a unique point $x_{0} \in C$ such that [14]

$$
d\left(x, x_{0}\right)=\inf \{d(x, y): y \in C\} .
$$

A point $x_{0}$ in $C$ is said to be a unique nearest point of $x \in E$. The metric projection of $E$ onto $C$ is the mapping $P_{C}: E \rightarrow C$ defined as: corresponding to each $x$ in $E$,

$$
P_{C}(x) \text { is the unique nearest point of } x \text { in } C \text {. }
$$

By Lemma 2.1 [13], for each $x, y \in E$ and $t \in[0,1]$, there exists a unique point $z \in[x, y]$ such that

$$
d(x, z)=(1-t) d(x, y) \text { and } d(y, z)=t d(x, y) .
$$

Throughout this paper, we shall use the notation $t x \oplus(1-t) y$ for the unique point $z$ satisfying (4). Finally, we give some known results in CAT(0) spaces needed in our results.

Lemma 1.0.1. [16] Let E be a CAT(0) space. Then

$$
d(k x \oplus(1-k) y, z) \leq k d(x, z)+(1-k) d(y, z)
$$

for all $x, y, z \in E$ and $k \in(0,1)$.

Lemma 1.0.2. [16] Let E be a CAT(0) space. Then

$$
d^{2}(k x \oplus(1-k) y, z) \leq k d^{2}(x, z)+(1-k) d^{2}(y, z)-k(1-k) d^{2}(x, y)
$$

for all $x, y, z \in E$ and $k \in(0,1)$. 
Lemma 1.0.3. [17] Let E be a CAT(O) space. Then

$$
d(k x \oplus(1-k) z, k y \oplus(1-k) z) \leq k d(x, y)
$$

for all $x, y, z \in E$ and $k \in(0,1)$.

Lemma 1.0.4. [18] Let E be a CAT(0) space. Then

$$
d(k x \oplus(1-k) z, m x \oplus(1-m) y) \leq|k-m| d(x, y)
$$

for all $x, y \in E$ and $k, m \in(0,1)$.

Lemma 1.0.5. [14] Let $E$ be a CAT(0) space, $p, q, r, s \in E$ and $k \in[0,1]$. Then

$$
d(k p \oplus(1-k) q, k r \oplus(1-k) s) \leq k d(p, r)+(1-k) d(q, s) .
$$

Berg and Nikolaev [19] introduced an important concept of quasilinearization as follows:

Denote a pair $(a, b) \in E \times E$ by $\overrightarrow{a b}$ and call it a vector. The quasilinearization is a map $\langle.,\rangle:. E^{2} \times E^{2} \rightarrow \mathbb{R}$ defined by

$$
\langle\overrightarrow{a b}, \overrightarrow{c d}\rangle=\frac{1}{2}\left(d^{2}(a, d)+d^{2}(b, c)-d^{2}(a, c)-d^{2}(b, d)\right)
$$

for all $a, b, c, d \in E$. One can easily see that $\langle\overrightarrow{a b}, \overrightarrow{c d}\rangle=\langle\overrightarrow{c d}, \overrightarrow{a b}\rangle,\langle\overrightarrow{a b}, \overrightarrow{c d}\rangle=-\langle\overrightarrow{b a}, \overrightarrow{c d}\rangle$ and $\langle\overrightarrow{a x}, \overrightarrow{c d}\rangle+$ $\langle\overrightarrow{x b}, \overrightarrow{c d}\rangle=\langle\overrightarrow{a b}, \overrightarrow{c d}\rangle$ for all $a, b, c, d, x \in E$. A metric space $E$ satisfies Cauchy-Schwarz inequality if

$$
|\langle\overrightarrow{a b}, \overrightarrow{c d}\rangle| \leq d(a, b) d(c, d)
$$

for all $a, b, c, d \in E$. It is known that a geodesically connected metric space is CAT(0) space if and only if it satisfies the Cauchy-Schwarz inequality [19].

Following are some important lemmas.

Lemma 1.0.6. [10] Let $E$ be a complete CAT(0) space. Then for all $u, x, y \in E$, the following inequality

$$
d^{2}(x, u) \leq d^{2}(y, u)+2\langle\overrightarrow{x y}, \overrightarrow{x u}\rangle
$$

holds.

Lemma 1.0.7. [10] Let $E$ be a complete CAT(0) space. If for any $u, v \in E$ and $t \in[0,1]$, we set $u_{t}=t u \oplus(1-t) v$. Then, for each $x, y \in E$, we have

1. $\left\langle\overrightarrow{u_{t} x}, \overrightarrow{u_{t} y}\right\rangle \leq t\left\langle\overrightarrow{u x}, \overrightarrow{u_{t} y}\right\rangle+(1-t)\left\langle\overrightarrow{v x}, \overrightarrow{u_{t} y}\right\rangle$;

2. $\left\langle\overrightarrow{u_{t} x}, \overrightarrow{u y}\right\rangle \leq t\langle\overrightarrow{u x}, \overrightarrow{u y}\rangle+(1-t)\langle\overrightarrow{v x}, \overrightarrow{u y}\rangle$;

3. $\left\langle\overrightarrow{u_{t} x}, \overrightarrow{v y}\right\rangle \leq t\langle\overrightarrow{u x}, \overrightarrow{v y}\rangle+(1-t)\langle\overrightarrow{v x}, \overrightarrow{v y}\rangle$.

Lemma 1.0.8. [20] Let $C$ be a nonempty convex subset of a complete CAT(O) space $E, x \in E$ and $u \in C$. Then

$$
u=P_{C} x \quad \text { if and only if }\langle\overrightarrow{y u}, \overrightarrow{u x}\rangle \geq 0 \quad \text { for all } y \in C .
$$

Now, we recall some convergence results in CAT(0) spaces.

Let $\left\{x_{n}\right\}$ be a bounded sequence in a $\operatorname{CAT}(0)$ space $E$. For $x \in E$, set

$$
r\left(x,\left\{x_{n}\right\}\right)=\limsup _{n \rightarrow \infty} d\left(x, x_{n}\right) .
$$

The asymptotic radius $r\left(\left\{x_{n}\right\}\right)$ of $\left\{x_{n}\right\}$ is given by

$$
r\left(\left\{x_{n}\right\}\right)=\inf \left\{r\left(x,\left\{x_{n}\right\}\right): x \in E\right\},
$$

and the asymptotic center $A\left(\left\{x_{n}\right\}\right)$ of $\left\{x_{n}\right\}$ is the set

$$
A\left(\left\{x_{n}\right\}\right)=\left\{x \in E: r\left(x,\left\{x_{n}\right\}\right)=r\left(\left\{x_{n}\right\}\right)\right\} .
$$


It is well known that in a $\operatorname{CAT}(0)$ space, $A\left(\left\{x_{n}\right\}\right)$ consists of exactly one point. A sequence $\left\{x_{n}\right\}$ is said to $\Delta$-convergent to $x \in E$ if $A\left(\left\{x_{n_{k}}\right\}\right)=\{x\}$ for every subsequence $\left\{x_{n_{k}}\right\}$ of $\left\{x_{n}\right\}$. The uniqueness of asymptotic center implies that the CAT(0) space satisfies the Opial's property, i.e., for given $\left\{x_{n}\right\} \subset E$ such that $\left\{x_{n}\right\}$ $\Delta$-converges to $x$ and given $y \in E$ with $y \neq x$,

$$
\limsup _{n \rightarrow \infty} d\left(x_{n}, x\right)<\limsup _{n \rightarrow \infty} d\left(x_{n}, y\right) .
$$

Lemma 1.0.9. [9] Every bounded sequence in a complete CAT(O) space has a $\Delta$-convergent subsequence.

Lemma 1.0.10. [9] Let $E$ be a CAT(O) space, $\left\{x_{n}\right\}$ a sequence in $E$ and $x \in E$. Then $\left\{x_{n}\right\} \Delta$-converges to $x$ if and only if $\lim \sup _{n \rightarrow \infty}\left\langle\overrightarrow{x_{n} x}, \overrightarrow{y x}\right\rangle \leq 0$ for all $y \in E$.

A multivalued nonexpansive operator $T: C \rightarrow K(C)$ satisfies the endpoint condition, if $F(T) \neq \phi$ and $T(x)=$ $\{x\}$ for any $x \in F(T)$.

Lemma 1.0.11. [11] Assume that $C$ is a closed convex subset of a complete CAT(O) space E. If $T: C \rightarrow C B(C)$ satisfies the endpoint condition, then $F(T)$ is closed and convex.

Lemma 1.0.12. [9] If $C$ is a closed convex subset of a complete $C A T(0)$ space $E$ and $T: C \rightarrow K(C)$ is a nonexpansive mapping, then the conditions $\left\{x_{n}\right\} \Delta$-converges to $x$ and $d\left(x_{n}, T x_{n}\right) \rightarrow 0$ imply $x \in F(T)$.

Recall that a continuous linear functional $\mu$ on $l_{\infty}$, the Banach space of bounded real sequence, is called a Banach limit if $\|\mu\|=\mu(1,1, \ldots)$ and $\mu_{n}\left(a_{n}\right)=\mu_{n}\left(a_{n+1}\right)$ for all $\left\{a_{n}\right\} \in l_{\infty}$. The following lemma is an important tool for proving the convergence of a sequence $\left\{d^{2}\left(x_{n}, q\right)\right\}$.

Lemma 1.0.13. [21] Let $\left\{a_{n}\right\}$ be a sequence of non-negative real number satisfying the property

$$
a_{n+1} \leq\left(1-\alpha_{n}\right) a_{n}+\alpha_{n} \beta_{n}, \quad n \geq 0,
$$

where $\left\{\alpha_{n}\right\} \subseteq(0,1)$ and $\left\{\beta_{n}\right\} \subseteq \mathbb{R}$ such that

1. $\sum_{n=0}^{\infty} \alpha_{n}=0$;

2. $\lim \sup _{n \rightarrow \infty} \beta_{n} \leq 0$ or $\sum_{n=0}^{\infty}\left|\alpha_{n} \beta_{n}\right|<\infty$.

Then $\left\{a_{n}\right\}$ converges to zero, as $n \rightarrow \infty$.

\section{Results}

Now, we present the following convergence result of our iterative scheme (2).

Theorem 2.0.1. Let $C$ be a nonempty closed convex subset of a complete CAT(O) space $E, T: C \rightarrow K(C) a$ multivalued nonexpansive mapping satisfying the endpoint condition and $f: C \rightarrow C$ a contraction with $k \in$ $(0,1)$. If sequences $\left\{\alpha_{n}\right\}$ and $\left\{\beta_{n}\right\} \in(0,1)$ satisfy

A1 $\lim _{n \rightarrow \infty} \alpha_{n}=0$,

A2 $\sum_{n=1}^{\infty} \alpha_{n}=\infty$,

A3 $\sum_{n=1}^{\infty}\left|\alpha_{n+1}-\alpha_{n}\right|=\infty$,

A4 $0<\epsilon \leq \beta_{n} \leq \beta_{n+1}<1$ for all $n \geq 0$.

Then the sequence generated by (2) converges strongly to a fixed point $x_{\star}$ of the nonexpansive mapping $T$, which solves the variational inequality

$$
\left\langle\overrightarrow{x_{\star} f\left(x_{\star}\right)}, \overrightarrow{x x_{\star}}\right\rangle \geq 0 \text { for all } x \in F(T) .
$$

That is, $\chi_{\star} \in P_{F(T)} f\left(\chi_{\star}\right)$.

Proof. We shall be divide the proof into four steps. 
STEP 1 First we show that the sequence $\left\{x_{n}\right\}$ is bounded. For any $p \in F(T)$, we have

$$
\begin{aligned}
d\left(x_{n+1}, q\right) & \leq d\left(\alpha_{n} f\left(x_{n}\right) \oplus\left(1-\alpha_{n}\right) u_{n}, q\right) \\
& \leq \alpha_{n} d\left(f\left(x_{n}\right), q\right)+\left(1-\alpha_{n}\right) d\left(u_{n}, q\right) \\
& \leq \alpha_{n} d\left(f\left(x_{n}\right), f(q)\right)+\alpha_{n} d(f(q), q)+\left(1-\alpha_{n}\right) d\left(u_{n}, T q\right) \\
& \leq \alpha_{n} d\left(f\left(x_{n}\right), f(q)\right)+\alpha_{n} d(f(q), q)+\left(1-\alpha_{n}\right) H\left(T y_{n}, T q\right) \\
& \leq k \alpha_{n} d\left(x_{n}, q\right)+\alpha_{n} d(f(q), q)+\left(1-\alpha_{n}\right) d\left(y_{n}, q\right) \\
& \leq k \alpha_{n} d\left(x_{n}, q\right)+\left(1-\alpha_{n}\right) d\left(\beta_{n} x_{n} \oplus\left(1-\beta_{n}\right) z_{n+1}, q\right)+\alpha_{n} d(f(q), q) \\
& \leq k \alpha_{n} d\left(x_{n}, q\right)+\left(1-\alpha_{n}\right)\left[\beta_{n} d\left(x_{n}, q\right)+\left(1-\beta_{n}\right) d\left(z_{n+1}, q\right)\right]+\alpha_{n} d(f(q), q) \\
& \left.\leq\left(k \alpha_{n}+\left(1-\alpha_{n}\right) \beta_{n}\right) d\left(x_{n}, q\right)+\left(1-\alpha_{n}\right)\left(1-\beta_{n}\right) d\left(z_{n+1}, T q\right)\right]+\alpha_{n} d(f(q), q) \\
& \left.\leq\left(k \alpha_{n}+\left(1-\alpha_{n}\right) \beta_{n}\right) d\left(x_{n}, q\right)+\left(1-\alpha_{n}\right)\left(1-\beta_{n}\right) H\left(T x_{n+1}, T q\right)\right]+\alpha_{n} d(f(q), q) \\
& \left.\leq\left(k \alpha_{n}+\left(1-\alpha_{n}\right) \beta_{n}\right) d\left(x_{n}, q\right)+\left(1-\alpha_{n}\right)\left(1-\beta_{n}\right) d\left(x_{n+1}, q\right)\right]+\alpha_{n} d(f(q), q) .
\end{aligned}
$$

Therefore,

$$
\left[1-\left(1-\alpha_{n}\right)\left(1-\beta_{n}\right)\right] d\left(x_{n+1}, q\right) \leq\left(k \alpha_{n}+\left(1-\alpha_{n}\right) \beta_{n}\right) d\left(x_{n}, q\right)+\alpha_{n} d(f(q), q) .
$$

Since $\alpha_{n}, \beta_{n} \in(0,1)$, we have $\left[1-\left(1-\alpha_{n}\right)\left(1-\beta_{n}\right)\right]<1$. Hence,

$$
\begin{aligned}
d\left(x_{n+1}, p\right) & \leq\left[\frac{k \alpha_{n}+\left(1-\alpha_{n}\right) \beta_{n}}{1-\left(1-\alpha_{n}\right)\left(1-\beta_{n}\right)}\right] d\left(x_{n}, q\right)+\frac{\alpha_{n}}{1-\left(1-\alpha_{n}\right)\left(1-\beta_{n}\right)} d(f(q), q) \\
& =\left[1-\frac{\alpha_{n}(1-k)}{1-\left(1-\alpha_{n}\right)\left(1-\beta_{n}\right)}\right] d\left(x_{n}, p\right)+\frac{\alpha_{n}(1-k)}{1-\left(1-\alpha_{n}\right)\left(1-\beta_{n}\right)}\left(\frac{1}{1-k} d(f(q), q)\right) .
\end{aligned}
$$

Consequently,

$$
d\left(x_{n+1}, q\right) \leq \max \left\{d\left(x_{n}, q\right), \frac{1}{1-k} d(f(q), q)\right\}
$$

By induction we obtain that

$$
d\left(x_{n+1}, q\right) \leq \max \left\{d\left(x_{0}, q\right), \frac{1}{1-k} d(f(q), q)\right\} .
$$

Thus $\left\{x_{n}\right\}$ is bounded. Furthermore, we deduce $f\left(x_{n}\right),\left\{u_{n}\right\}$ and $\left\{z_{n+1}\right\}$ are bounded.

STEP 2 Now, we prove that $\lim _{n \rightarrow \infty} d\left(x_{n+1}, x_{n}\right)=0$. By (2) and Lemmas 1.0.3 and 1.0.4, we have

$$
\begin{aligned}
d\left(y_{n}, y_{n-1}\right)= & d\left(\beta_{n} x_{n} \oplus\left(1-\beta_{n}\right) z_{n+1}, \beta_{n-1} x_{n-1} \oplus\left(1-\beta_{n-1}\right) z_{n}\right) \\
\leq & d\left(\beta_{n} x_{n} \oplus\left(1-\beta_{n}\right) z_{n+1}, \beta_{n} x_{n} \oplus\left(1-\beta_{n}\right) z_{n}\right)+d\left(\beta_{n} x_{n} \oplus\left(1-\beta_{n}\right) z_{n}, \beta_{n} x_{n-1} \oplus\left(1-\beta_{n}\right) z_{n}\right) \\
& +d\left(\beta_{n} x_{n-1} \oplus\left(1-\beta_{n}\right) z_{n}, \beta_{n-1} x_{n-1} \oplus\left(1-\beta_{n-1}\right) z_{n}\right) \\
\leq & \left(1-\beta_{n}\right) d\left(z_{n}, z_{n+1}\right)+\beta_{n} d\left(x_{n-1}, x_{n}\right)+\left|\beta_{n}-\beta_{n-1}\right| d\left(x_{n-1}, z_{n}\right) \\
\leq & \left(1-\beta_{n}\right) d\left(x_{n}, x_{n+1}\right)+\beta_{n} d\left(x_{n-1}, x_{n}\right)+\left|\beta_{n}-\beta_{n-1}\right| d\left(x_{n-1}, x_{n}\right) \\
\leq & \left(1-\beta_{n}\right) d\left(x_{n}, x_{n+1}\right)+\beta_{n-1} d\left(x_{n-1}, x_{n}\right)
\end{aligned}
$$

Again using Lemmas 1.0.3 and 1.0.4 and inequality (10), we obtain that

$$
\begin{aligned}
d\left(x_{n+1}, x_{n}\right)= & d\left(\alpha_{n} f\left(x_{n}\right) \oplus\left(1-\alpha_{n}\right) u_{n}, \alpha_{n-1} f y_{n-1} \oplus\left(1-\alpha_{n-1}\right) u_{n-1}\right) \\
\leq & d\left(\alpha_{n} f\left(x_{n}\right) \oplus\left(1-\alpha_{n}\right) u_{n}, \alpha_{n} f x_{n} \oplus\left(1-\alpha_{n}\right) u_{n-1}\right) \\
& +d\left(\alpha_{n} f\left(x_{n}\right) \oplus\left(1-\alpha_{n}\right) u_{n-1}, \alpha_{n} f\left(x_{n-1}\right) \oplus\left(1-\alpha_{n}\right) u_{n-1}\right) \\
& +d\left(\alpha_{n} f\left(x_{n-1}\right) \oplus\left(1-\alpha_{n}\right) u_{n-1}, \alpha_{n-1} f\left(x_{n-1}\right) \oplus\left(1-\alpha_{n-1}\right) u_{n-1}\right) \\
\leq & \left(1-\alpha_{n}\right) d\left(u_{n}, u_{n-1}\right)+\alpha_{n} d\left(f\left(x_{n}\right), f\left(x_{n-1}\right)\right)+\left|\alpha_{n}-\alpha_{n-1}\right| d\left(f\left(x_{n-1}\right), u_{n-1}\right) \\
\leq & \left(1-\alpha_{n}\right) d\left(y_{n}, y_{n-1}\right)+k \alpha_{n} d\left(x_{n}, x_{n-1}\right)+\left|\alpha_{n}-\alpha_{n-1}\right| M
\end{aligned}
$$




$$
\begin{aligned}
& \leq\left(1-\beta_{n}\right)\left(1-\alpha_{n}\right) d\left(x_{n}, x_{n+1}\right)+\left|\beta_{n-1}\left(1-\alpha_{n}\right) d\left(x_{n-1}, x_{n}\right)+k \alpha_{n} d\left(x_{n-1}, x_{n}\right)+\right| \alpha_{n}-\alpha_{n-1} \mid M \\
& =\left(1-\beta_{n}\right)\left(1-\alpha_{n}\right) d\left(x_{n}, x_{n+1}\right)+\left(\beta_{n-1}\left(1-\alpha_{n}\right)+k \alpha_{n}\right) d\left(x_{n-1}, x_{n}\right)+\left|\alpha_{n}-\alpha_{n-1}\right| M
\end{aligned}
$$

where $M \geq \sup _{n \geq 0} d\left(f\left(x_{n-1}\right), u_{n-1}\right)$. Therefore

$$
\left(1-\left(1-\alpha_{n}\right)\right)\left(1-\beta_{n}\right) d\left(x_{n+1}, x_{n}\right) \leq\left(\beta_{n-1}\left(1-\alpha_{n}\right)+k \alpha_{n}\right) d\left(x_{n-1}, x_{n}\right)+\left|\alpha_{n}-\alpha_{n-1}\right| M .
$$

Thus,

$$
\begin{aligned}
d\left(x_{n+1}, x_{n}\right) & \leq \frac{\beta_{n-1}\left(1-\alpha_{n}\right)+k \alpha_{n}}{1-\left(1-\alpha_{n}\right)\left(1-\beta_{n}\right)} d\left(x_{n-1}, x_{n}\right)+\frac{\left|\alpha_{n}-\alpha_{n-1}\right|}{\left.\left.1-\left(1-\alpha_{n}\right)\left(1-\beta_{n}\right)\right)\left(1-\beta_{n}\right)\right]} M \\
& \leq\left[1-\frac{\left.\alpha_{n}(1-k)+\left(\beta_{n}-\beta_{n-1}\right)\left(1-\alpha_{n}\right)\right)}{1-\left(1-\alpha_{n}\right)\left(1-\beta_{n}\right)}\right] \times d\left(x_{n-1}, x_{n}\right)+\frac{\left|\alpha_{n}-\alpha_{n-1}\right|}{1-\left(1-\alpha_{n}\right)\left(1-\beta_{n}\right)} M .
\end{aligned}
$$

Clearly, from condition (A4), we have

$$
0<\epsilon \leq 1-\left(1-\alpha_{n}\right)\left(1-\beta_{n}\right)<1 \text {. }
$$

This implies that

$$
\frac{\left.\alpha_{n}(1-k)+\left(\beta_{n}-\beta_{n-1}\right)\left(1-\alpha_{n}\right)\right)}{1-\left(1-\alpha_{n}\right)\left(1-\beta_{n}\right)} \geq \alpha_{n}(1-k)-\left(\beta_{n}-\beta_{n-1}\right) \geq \alpha_{n}(1-k) .
$$

Therefore,

$$
d\left(x_{n+1}, x_{n}\right) \leq \alpha_{n}(1-k) d\left(x_{n-1}, x_{n}\right)+\left|\alpha_{n}-\alpha_{n-1}\right| \frac{M}{\epsilon} .
$$

It follows from conditions (A1),(A2) and Lemma 1.0.13 that

$$
d\left(x_{n+1}, x_{n}\right) \rightarrow 0 \text { as } n \rightarrow \infty \text {. }
$$

Now, we prove that $\lim _{n \rightarrow \infty} d\left(x_{n}, T_{n}\right)=0$. By definition (2), we have $d\left(x_{n}, z_{n+1}\right) \leq d\left(x_{n}, x_{n+1}\right)$. Thus

$$
\begin{aligned}
d\left(x_{n}, z_{n}\right) & \leq d\left(x_{n}, z_{n+1}\right)+d\left(z_{n+1}, z_{n}\right) \\
& \leq d\left(x_{n}, x_{n+1}\right)+d\left(x_{n}, x_{n+1}\right) .
\end{aligned}
$$

Using the fact that $\lim _{n \rightarrow \infty} d\left(x_{n}, x_{n+1}\right)=0$, we have $\lim _{n \rightarrow \infty} d\left(x_{n}, z_{n}\right)=0$. Consequently, $\lim _{n \rightarrow \infty} d\left(x_{n}, T x_{n}\right)=$ 0 . Note that

$$
\begin{aligned}
d\left(x_{n}, T y_{n}\right) & \leq d\left(x_{n}, u_{n}\right) \\
& \leq d\left(x_{n}, x_{n+1}\right)+d\left(x_{n+1}, u_{n}\right) \\
& \leq d\left(x_{n}, x_{n+1}\right)+\alpha_{n} d\left(f\left(x_{n}\right), u_{n}\right) \\
& \leq d\left(x_{n}, x_{n+1}\right)+\alpha_{n} M_{1}
\end{aligned}
$$

where $M_{1} \geq \sup _{n \geq 0} d\left(f\left(x_{n}\right), u_{n}\right)$ for all $n \geq 0$. Then by condition (A1) and $\lim _{n \rightarrow \infty} d\left(x_{n}, x_{n+1}\right)=0$, we obtain that $\lim _{n \rightarrow \infty} d\left(x_{n}, T y_{n}\right)=0$.

STEP 3 Now, we show that $\left\{x_{n}\right\}$ converges strongly to $x_{\star}$ where $x_{\star}=P_{F(T)} f\left(x_{\star}\right)$ which is equivalent to variational inequality (32).

Since $\left\{x_{n}\right\}$ is bounded, by Lemma 1.0.9, there exists a subsequence $\left\{x_{n_{j}}\right\}$ of $\left\{x_{n}\right\}$ such that $\Delta-\lim _{j \rightarrow \infty} x_{n_{j}}=x_{\star}$. Also,

$$
\limsup _{n \rightarrow \infty}\left\langle\overrightarrow{x_{\star} f\left(\chi_{\star}\right)}, \overrightarrow{\left.\chi_{\star} \chi_{n}\right)}\right\rangle=\limsup _{j \rightarrow \infty}\left\langle\overrightarrow{x_{\star} f\left(\chi_{\star}\right)}, \overrightarrow{\left.\chi_{\star} \chi_{n_{j}}\right)}\right\rangle
$$

From Lemma 1.0.12, we get $x_{\star} \in F(T)$. Again, by Lemma 1.0.10, we have

$$
\limsup _{j \rightarrow \infty}\left\langle\overrightarrow{x_{\star} f\left(x_{\star}\right)}, \overrightarrow{\left.x_{\star} x_{j}\right)}\right\rangle \leq 0
$$


By (13) and (15) we have

$$
\limsup _{n \rightarrow \infty}\left\langle\overrightarrow{\chi \star f\left(x_{\star}\right)}, \overrightarrow{\left.x_{\star} \chi_{n}\right)}\right\rangle \leq 0 .
$$

We now prove that $\left\{x_{n}\right\}$ converges strongly to $x_{\star}$. If we set $v_{n}=\alpha_{n} x_{\star} \oplus\left(1-\alpha_{n}\right) u_{n}$ where $u_{n} \in T y_{n}$. Then from Lemma 1.0.6 and 1.0.7, we have

$$
\begin{aligned}
& d^{2}\left(x_{n+1}, x_{\star}\right) \leq d^{2}\left(v_{n}, x_{\star}\right)+2\left\langle\overrightarrow{x_{n+1} v_{n}}, \overrightarrow{x_{n+1} \chi_{\star}}\right\rangle \\
& \leq d^{2}\left(\alpha_{n} \chi_{\star} \oplus\left(1-\alpha_{n}\right) u_{n}, x_{\star}\right)+2\left\langle\overrightarrow{x_{n+1} v_{n}}, \overrightarrow{x_{n+1} x_{\star}}\right\rangle \\
& \leq\left[\alpha_{n} d\left(x_{\star}, x_{\star}\right)+\left(1-\alpha_{n}\right) d\left(u_{n}, x_{\star}\right)\right]^{2}+2\left[\alpha_{n}\left\langle\overrightarrow{f\left(x_{n}\right) v_{n}}, \overrightarrow{x_{n+1} X_{\star}}\right\rangle+\left(1-\alpha_{n}\right)\left\langle\overrightarrow{u_{n} v_{n}}, \overrightarrow{x_{n+1} x_{\star}}\right\rangle\right] \\
& \leq\left(1-\alpha_{n}\right)^{2} d\left(u_{n}, x_{\star}\right)+2\left[\alpha_{n} \alpha_{n}\left\langle\overrightarrow{f\left(x_{n}\right) x_{\star}}, \overrightarrow{x_{n+1} x_{\star}}\right\rangle+\alpha_{n}\left(1-\alpha_{n}\right)\left\langle\overrightarrow{f\left(x_{n}\right) u_{n}}, \overrightarrow{x_{n+1} x_{\star}}\right\rangle\right. \\
& \left.+\alpha_{n}\left(1-\alpha_{n}\right)\left\langle\overrightarrow{u_{n} X_{\star}}, \overrightarrow{x_{n+1} X_{\star}}\right\rangle+\left(1-\alpha_{n}\right)^{2}\left\langle\overrightarrow{u_{n} u_{n}}, \overrightarrow{x_{n+1} X_{\star}}\right\rangle\right] \\
& \leq\left(1-\alpha_{n}\right)^{2} d\left(y_{n}, x_{\star}\right)+2\left[\alpha_{n}^{2}\left\langle\overrightarrow{f\left(x_{n}\right) x_{\star}}, \overrightarrow{x_{n+1} x_{\star}}\right\rangle+\alpha_{n}\left(1-\alpha_{n}\right)\left\langle\overrightarrow{f\left(x_{n}\right) x_{\star}}, \overrightarrow{x_{n+1} x_{\star}}\right\rangle\right] \\
& =\left(1-\alpha_{n}\right)^{2} d\left(y_{n}, x_{\star}\right)+2 \alpha_{n}\left\langle\overrightarrow{f\left(x_{n}\right) x_{\star}}, \overrightarrow{x_{n+1} X_{\star}}\right\rangle
\end{aligned}
$$

Note that

$$
\left\langle\overrightarrow{f\left(x_{n}\right) \chi_{\star}}, \overrightarrow{x_{n+1} X_{\star}}\right\rangle=\left(1-\alpha_{n}\right)\left\langle\overrightarrow{u_{n} X_{\star}}, \overrightarrow{f\left(x_{n}\right) \chi_{\star}}\right\rangle .
$$

By Cauchy Schwarz inequality, (16) becomes

$$
\begin{aligned}
d^{2}\left(x_{n+1}, x_{\star}\right) & \leq\left(1-\alpha_{n}\right)^{2} d^{2}\left(y_{n}, x_{\star}\right)+2 \alpha_{n}\left(1-\alpha_{n}\right)\left\langle\overrightarrow{u_{n} x_{\star}}, \overrightarrow{f\left(x_{n}\right) x_{\star}}\right\rangle \\
& \left.\left.\leq\left(1-\alpha_{n}\right)^{2} d^{2}\left(y_{n}, x_{\star}\right)+2 \alpha_{n}\left(1-\alpha_{n}\right)\left\langle\overrightarrow{f\left(x_{n}\right) f\left(x_{\star}\right.}\right), \overrightarrow{u_{n} \chi_{\star}}\right\rangle+2 \alpha_{n}\left(1-\alpha_{n}\right)\left\langle\overrightarrow{f\left(x_{\star}\right.}\right) x_{\star}, \overrightarrow{u_{n} x_{\star}}\right\rangle \\
& \leq\left(1-\alpha_{n}\right)^{2} d^{2}\left(y_{n}, x_{\star}\right)+2 \alpha_{n}\left(1-\alpha_{n}\right)\left\langle\overrightarrow{f\left(x_{n}\right) f\left(x_{\star}\right)}, \overrightarrow{u_{n} \chi_{\star}}\right\rangle+\gamma_{n} \\
& \leq\left(1-\alpha_{n}\right)^{2} d^{2}\left(y_{n}, x_{\star}\right)+2 \alpha_{n}\left(1-\alpha_{n}\right) d\left(f\left(x_{n}\right), f\left(x_{\star}\right)\right) d\left(u_{n}, x_{\star}\right)+\gamma_{n} \\
& \leq\left(1-\alpha_{n}\right)^{2} d^{2}\left(y_{n}, x_{\star}\right)+2 \alpha_{n}\left(1-\alpha_{n}\right) k d\left(x_{n}, x_{\star}\right) d\left(y_{n}, x_{\star}\right)+\gamma_{n}
\end{aligned}
$$

where

$$
\left.\gamma_{n}=2 \alpha_{n}\left(1-\alpha_{n}\right)\left\langle\overrightarrow{f\left(\chi_{\star}\right)}\right)_{\star}, \overrightarrow{u_{n} \chi_{\star}}\right\rangle
$$

Then,

$$
0 \leq\left(1-\alpha_{n}\right)^{2} d\left(y_{n}, x_{\star}\right)+2 \alpha_{n}\left(1-\alpha_{n}\right) k d\left(x_{n}, x_{\star}\right) d^{2}\left(y_{n}, x_{\star}\right)+\gamma_{n}-d^{2}\left(x_{n+1}, x_{\star}\right) .
$$

By using the quadratic formula to solve the above quadratic equation for $d^{2}\left(y_{n}, x_{\star}\right)$, we get

$$
\begin{aligned}
d\left(y_{n}, x_{\star}\right) & \geq \frac{1}{2\left(1-\alpha_{n}\right)^{2}}\left(-2 k \alpha_{n}\left(1-\alpha_{n}\right) d\left(x_{n}, x_{\star}\right)+\sqrt{\left(2 \alpha_{n}\left(1-\alpha_{n}\right) k\right)^{2}-4\left(1-\alpha_{n}\right)^{2}\left(\gamma_{n}-d^{2}\left(x_{n+1}, x_{\star}\right)\right)}\right) \\
& =\left(-k \alpha_{n} d\left(x_{n}, x_{\star}\right)\right) \times \frac{\sqrt{k^{2} \alpha_{n}^{2} d^{2}\left(x_{n}, x_{\star}\right)-\left(\gamma_{n}-d^{2}\left(x_{n+1}, x_{\star}\right)\right)}}{1-\alpha_{n}} .
\end{aligned}
$$

Moreover,

$$
d\left(\beta_{n} x_{n} \oplus\left(1-\beta_{n}\right) z_{n+1}, x_{\star}\right) \geq \frac{-k \alpha_{n} d\left(x_{n}, x_{\star}\right) \sqrt{k^{2} \alpha_{n}^{2} d^{2}\left(x_{n}, x_{\star}\right)-\left(\gamma_{n}-d^{2}\left(x_{n+1}, x_{\star}\right)\right)}}{1-\alpha_{n}}
$$

implies that

$$
\beta_{n} d\left(x_{n}, x_{\star}\right)+\left(1-\beta_{n}\right) d\left(z_{n+1}, x_{\star}\right) \geq \frac{-k \alpha_{n} d\left(x_{n}, x_{\star}\right) \sqrt{k^{2} \alpha_{n}^{2} d^{2}\left(x_{n}, x_{\star}\right)-\left(\gamma_{n}-d^{2}\left(x_{n+1}, x_{\star}\right)\right)}}{1-\alpha_{n}} .
$$

Now

$$
\begin{aligned}
\left(1-\alpha_{n}\right) \beta_{n} d\left(x_{n}, \chi_{\star}\right) & +\left(1-\alpha_{n}\right)\left(1-\beta_{n}\right) d\left(z_{n+1}, x_{\star}\right) \\
\geq & -k \alpha_{n} d\left(x_{n}, x_{\star}\right) \sqrt{k^{2} \alpha_{n}^{2} d^{2}\left(x_{n}, x_{\star}\right)-\left(\gamma_{n}-d^{2}\left(x_{n+1}, x_{\star}\right)\right)},
\end{aligned}
$$


implies that

$$
\left(\left(1-\alpha_{n}\right) \beta_{n}-k \alpha_{n}\right) d\left(x_{n}, x_{\star}\right)+\left(1-\alpha_{n}\right)\left(1-\beta_{n}\right) d\left(z_{n+1}, x_{\star}\right) \geq \sqrt{k^{2} \alpha_{n}^{2} d^{2}\left(x_{n}, \chi_{\star}\right)-\left(\gamma_{n}-d^{2}\left(x_{n+1}, x_{\star}\right)\right)} .
$$

Squaring both sides in the above inequality and using the following inequality

$$
2 d\left(x_{n}, x_{\star}\right) d\left(x_{n+1}, x_{\star}\right) \leq d^{2}\left(x_{n}, x_{\star}\right)+d^{2}\left(x_{n+1}, x_{\star}\right)
$$

we obtain that

$$
\begin{aligned}
k^{2} \alpha_{n}^{2} d^{2}\left(x_{n}, \chi_{\star}\right)-\gamma_{n}+d^{2}\left(x_{n+1}, \chi_{\star}\right) \leq & \left(k \alpha_{n}+\left(1-\alpha_{n}\right) \beta_{n}\right)^{2} d^{2}\left(x_{n}, x_{\star}\right)+\left(1-\beta_{n}\right)^{2}\left(1-\alpha_{n}\right)^{2} d^{2}\left(x_{n+1}, \chi_{\star}\right) \\
& +2\left(k \alpha_{n}+\left(1-\alpha_{n}\right) \beta_{n}\right)\left(1-\beta_{n}\right)\left(1-\alpha_{n}\right) \times d\left(x_{n}, \chi_{\star}\right) d\left(x_{n+1}, \chi_{\star}\right) \\
\leq & \left(k \alpha_{n}+\left(1-\alpha_{n}\right) \beta_{n}\right)^{2} d^{2}\left(x_{n}, x_{\star}\right)+\left(1-\beta_{n}\right)^{2}\left(1-\alpha_{n}\right)^{2} d^{2}\left(x_{n+1}, x_{\star}\right) \\
& +\left(k \alpha_{n}+\left(1-\alpha_{n}\right) \beta_{n}\right)\left(1-\beta_{n}\right)\left(1-\alpha_{n}\right) \times\left(d^{2}\left(x_{n}, x_{\star}\right)+d^{2}\left(x_{n+1}, x_{\star}\right)\right) .
\end{aligned}
$$

This gives

$$
\begin{aligned}
\left.\left(1-\left(1-\beta_{n}\right)^{2}\left(1-\alpha_{n}\right)^{2}-\left(k \alpha_{n}+\left(1-\alpha_{n}\right) \beta_{n}\right)\left(1-\beta_{n}\right)\left(1-\alpha_{n}\right)\right) d^{2}\left(x_{n+1}, \chi_{\star}\right)\right) & \\
\leq & \left(\left(k \alpha_{n}+\left(1-\alpha_{n}\right) \beta_{n}\right)^{2}+\left(k \alpha_{n}+\left(1-\alpha_{n}\right) \beta_{n}\right)\left(1-\beta_{n}\right)\left(1-\alpha_{n}\right)\right) d^{2}\left(x_{n}, x_{\star}\right)+\gamma_{n}
\end{aligned}
$$

which can be further simplified as

$$
\begin{aligned}
\left(1-\left(1-\beta_{n}\right)\left(1-\alpha_{n}\right)\right. & \left.\left(1+(k-1) \alpha_{n}\right) d^{2}\left(x_{n+1}, x_{\star}\right)\right) \\
& \leq\left(\left(k \alpha_{n}+\left(1-\alpha_{n}\right)\left(1+(k-1) \alpha_{n}\right)-k^{2} \alpha_{n}^{2}\right) d^{2}\left(x_{n}, x_{\star}\right)+\gamma_{n}\right.
\end{aligned}
$$

Therefore,

$$
\left.d^{2}\left(x_{n+1}, x_{\star}\right)\right) \leq \frac{\left(\left(k \alpha_{n}+\left(1-\alpha_{n}\right)\left(1+(k-1) \alpha_{n}\right)-k^{2} \alpha_{n}^{2}\right)\right.}{\left(1-\left(1-\beta_{n}\right)\left(1-\alpha_{n}\right)\left(1+(k-1) \alpha_{n}\right)\right.} d^{2}\left(x_{n}, x_{\star}\right)+\frac{\gamma_{n}}{\left(1-\left(1-\beta_{n}\right)\left(1-\alpha_{n}\right)\left(1+(k-1) \alpha_{n}\right)\right.}
$$

Suppose that

$$
\xi_{n}=\frac{1}{\alpha_{n}}\left(1-\frac{\left(\left(k \alpha_{n}+\left(1-\alpha_{n}\right)\left(1+(k-1) \alpha_{n}\right)-k^{2} \alpha_{n}^{2}\right)\right.}{1-\left(1-\beta_{n}\right)\left(1-\alpha_{n}\right)\left(1+(k-1) \alpha_{n}\right)}\right)=\frac{2(1-k)+\alpha_{n}(2 k-1)}{1-\left(1-\beta_{n}\right)\left(1-\alpha_{n}\right)\left(1+(k-1) \alpha_{n}\right)} .
$$

From condition (A4), the sequence $\left\{\beta_{n}\right\}$ satisfies $0<\epsilon \leq \beta_{n} \leq \beta_{n+1}<1$ for all $n \geq 0$, and hence $\lim _{n \rightarrow \infty} \beta_{n}$ exists. Let $\lim _{n \rightarrow \infty} \beta_{n}=\beta>0$. Then

$$
\lim _{n \rightarrow \infty} \xi_{n}=\frac{2(1-k)}{\beta}>0
$$

Suppose $\delta_{0}>0$ satisfies

$$
\delta_{0}<\frac{2(1-k)}{\beta}
$$

then there exists $n_{0}$ such that $\xi_{n}>\delta_{0}$ for all $n \geq n_{0}$. Therefore, for all $n \geq n_{0}$, we have

$$
\frac{\left(\left(k \alpha_{n}+\left(1-\alpha_{n}\right)\left(1+(k-1) \alpha_{n}\right)-k^{2} \alpha_{n}^{2}\right)\right.}{1-\left(1-\beta_{n}\right)\left(1-\alpha_{n}\right)\left(1+(k-1) \alpha_{n}\right)} \leq 1-\delta_{0} \alpha_{n} .
$$

Then from equation (19), we have

$$
d^{2}\left(x_{n+1}, x_{\star}\right) \leq\left(1-\delta_{0} \alpha_{n}\right) d^{2}\left(x_{n}, x_{\star}\right)+\eta_{n}
$$

where $\eta_{n}=\frac{\gamma_{n}}{1-\left(1-\beta_{n}\right)\left(1-\alpha_{n}\right)\left(1+(k-1) \alpha_{n}\right)}$. From equations (18) and (15), we have

$$
\limsup _{n \rightarrow \infty} \frac{\gamma_{n}}{\alpha_{n}} \leq 0
$$

which further implies that

$$
\limsup _{n \rightarrow \infty} \frac{\eta_{n}}{\alpha_{n}} \leq 0
$$


From conditions (A1), (A2), (20), (21) and Lemma 1.0.13, we obtain

$$
d\left(x_{n}, x_{\star}\right)=0 \text { as } n \rightarrow \infty .
$$

Thus, $\left\{x_{n}\right\}$ converges strongly to $x_{\star}$.

STEP 4 Next, we show that $x_{\star}=P_{F}(T) f\left(x_{\star}\right)$. Let $q \in F(T)$. Since $T(x)$ is compact for any $x \in C, T(x) \in C B(X)$. From Lemma 1.0.12 it follows that $F(T)$ is closed and convex, which further implies that $P_{F(T)} z$ is well defined for any $z \in E$. Moreover, applying Lemma 1.0.2 we obtain that

$$
\begin{aligned}
d^{2}\left(x_{n+1}, q\right) \leq & d^{2}\left(\alpha_{n} f\left(x_{n}\right) \oplus\left(1-\alpha_{n}\right) u_{n}, q\right) \\
\leq & \alpha_{n} d^{2}\left(f\left(x_{n}\right), q\right)+\left(1-\alpha_{n}\right) d^{2}\left(u_{n}, q\right)-\alpha_{n}\left(1-\alpha_{n}\right) d^{2}\left(f\left(x_{n}\right), u_{n}\right) \\
\leq & \alpha_{n} d^{2}\left(f\left(x_{n}\right), q\right)+\left(1-\alpha_{n}\right) d^{2}\left(u_{n}, T q\right)-\alpha_{n}\left(1-\alpha_{n}\right) d^{2}\left(f\left(x_{n}\right), u_{n}\right) \\
\leq & \alpha_{n} d^{2}\left(f\left(x_{n}\right), q\right)+\left(1-\alpha_{n}\right) H^{2}\left(T y_{n}, T q\right)-\alpha_{n}\left(1-\alpha_{n}\right) d^{2}\left(f\left(x_{n}\right), u_{n}\right) \\
\leq & \alpha_{n} d^{2}\left(f\left(x_{n}\right), q\right)+\left(1-\alpha_{n}\right) d^{2}\left(y_{n}, q\right)-\alpha_{n}\left(1-\alpha_{n}\right) d^{2}\left(f\left(x_{n}\right), u_{n}\right) \\
\leq & \alpha_{n} d^{2}\left(f\left(x_{n}\right), q\right)+\left(1-\alpha_{n}\right)\left(\beta_{n} d^{2}\left(x_{n}, q\right)+\left(1-\beta_{n}\right) d^{2}\left(z_{n+1}, q\right)-\beta_{n}\left(1-\beta_{n}\right) d\left(x_{n}, z_{n+1}\right)\right) \\
& -\alpha_{n}\left(1-\alpha_{n}\right) d^{2}\left(f\left(x_{n}\right), u_{n}\right) \\
\leq & \alpha_{n} d^{2}\left(f\left(x_{n}\right), q\right)+\beta_{n}\left(1-\alpha_{n}\right) d^{2}\left(x_{n}, q\right) \\
& +\left(1-\beta_{n}\right)\left(1-\alpha_{n}\right) d^{2}\left(z_{n+1}, T q\right)-\beta_{n}\left(1-\beta_{n}\right) d\left(x_{n}, z_{n+1}\right)-\alpha_{n}\left(1-\alpha_{n}\right) d^{2}\left(f\left(x_{n}\right), u_{n}\right) \\
\leq & \alpha_{n} d^{2}\left(f\left(x_{n}\right), q\right)+\beta_{n}\left(1-\alpha_{n}\right) d^{2}\left(x_{n}, q\right) \\
& +\left(1-\beta_{n}\right)\left(1-\alpha_{n}\right) H^{2}\left(T x_{n+1}, T q\right)-\beta_{n}\left(1-\beta_{n}\right) d\left(x_{n}, z_{n+1}\right)-\alpha_{n}\left(1-\alpha_{n}\right) d^{2}\left(f\left(x_{n}\right), u_{n}\right) \\
\leq & \alpha_{n} d^{2}\left(f\left(x_{n}\right), q\right)+\beta_{n}\left(1-\alpha_{n}\right) d^{2}\left(x_{n}, q\right) \\
& +\left(1-\beta_{n}\right)\left(1-\alpha_{n}\right) d^{2}\left(x_{n+1}, q\right)-\beta_{n}\left(1-\beta_{n}\right) d\left(x_{n}, z_{n+1}\right)-\alpha_{n}\left(1-\alpha_{n}\right) d^{2}\left(f\left(x_{n}\right), u_{n}\right) .
\end{aligned}
$$

Applying the Banach limit, we have

$$
\begin{aligned}
\mu_{n} d^{2}\left(x_{n+1}, q\right) \leq & \mu_{n} \alpha_{n} d^{2}\left(f\left(x_{n}\right), q\right)+\mu_{n} \beta_{n}\left(1-\alpha_{n}\right) d^{2}\left(x_{n}, q\right) \\
& +\mu_{n}\left(1-\beta_{n}\right)\left(1-\alpha_{n}\right) d^{2}\left(x_{n+1}, q\right)-\mu_{n} \beta_{n}\left(1-\beta_{n}\right) d\left(x_{n}, z_{n+1}\right)-\mu_{n} \alpha_{n}\left(1-\alpha_{n}\right) d^{2}\left(f\left(x_{n}\right), u_{n}\right) .
\end{aligned}
$$

Therefore,

$$
\mu_{n} d^{2}\left(x_{n+1}, q\right) \leq \mu_{n} d^{2}\left(f\left(x_{n}\right), q\right)-\mu_{n} d^{2}\left(f\left(x_{n}\right), u_{n}\right) \leq \mu_{n} d^{2}\left(f\left(x_{n}\right), q\right)-\mu_{n} d^{2}\left(f\left(x_{n}\right), T y_{n}\right) .
$$

For any $\chi_{\star} \in C$ such that $x_{n} \rightarrow x_{\star}$, we obtain

$$
\begin{aligned}
d\left(f\left(x_{n}\right), T y_{n}\right) & \leq d\left(f\left(x_{n}\right), f\left(x_{\star}\right)\right)+d\left(f\left(x_{\star}\right), x_{\star}\right)+d\left(x_{\star}, T y_{n}\right) \\
& \leq k d\left(x_{n}, x_{\star}\right)+d\left(f\left(x_{\star}\right), x_{\star}\right)+H\left(T y_{n}, T x_{\star}\right) \\
& \leq k d\left(x_{n}, x_{\star}\right)+d\left(f\left(x_{\star}\right), x_{\star}\right)+d\left(y_{n}, x_{\star}\right) \\
& \leq k d\left(x_{n}, x_{\star}\right)+d\left(f\left(x_{\star}\right), x_{\star}\right)+\beta_{n} d\left(x_{n}, x_{\star}\right)+\left(1-\beta_{n}\right) d\left(x_{\star}, z_{n+1}\right) \\
& \leq k d\left(x_{n}, x_{\star}\right)+d\left(f\left(x_{\star}\right), x_{\star}\right)+\beta_{n} d\left(x_{n}, x_{\star}\right)+\left(1-\beta_{n}\right) d\left(T x_{\star}, z_{n+1}\right) \\
& \leq k d\left(x_{n}, x_{\star}\right)+d\left(f\left(x_{\star}\right), x_{\star}\right)+\beta_{n} d\left(x_{n}, x_{\star}\right)+\left(1-\beta_{n}\right) H\left(T x_{\star}, T x_{n+1}\right) \\
& \leq k d\left(x_{n}, x_{\star}\right)+d\left(f\left(x_{\star}\right), x_{\star}\right)+\beta_{n} d\left(x_{n}, x_{\star}\right)+\left(1-\beta_{n}\right) d\left(x_{\star}, x_{n+1}\right) \\
& =\left(k+\beta_{n}\right) d\left(x_{n}, x_{\star}\right)+d\left(f\left(x_{\star}\right), x_{\star}\right)+\left(1-\beta_{n}\right) d\left(x_{\star}, x_{n+1}\right)
\end{aligned}
$$

and

$$
\begin{aligned}
d\left(f x_{\star}, x_{\star}\right) & \leq d\left(f x_{\star}, f\left(x_{n}\right)\right)+d\left(f\left(x_{n}\right), T y_{n}\right)+d\left(T y_{n}, x_{\star}\right) \\
& \leq d\left(f x_{\star}, f\left(x_{n}\right)\right)+d\left(f\left(x_{n}\right), T y_{n}\right)+H\left(T y_{n}, T x_{\star}\right)
\end{aligned}
$$




$$
\begin{aligned}
& \leq k d\left(x_{n}, x_{\star}\right)+d\left(f\left(x_{n}\right), T y_{n}\right)+d\left(y_{n}, x_{\star}\right) \\
& \leq k d\left(x_{n}, x_{\star}\right)+d\left(f\left(x_{n}\right), T y_{n}\right)+\beta_{n} d\left(x_{n}, x_{\star}\right)+\left(1-\beta_{n}\right) d\left(z_{n+1}, x_{\star}\right) \\
& \leq k d\left(x_{n}, x_{\star}\right)+d\left(f\left(x_{n}\right), T y_{n}\right)+\beta_{n} d\left(x_{n}, x_{\star}\right)+\left(1-\beta_{n}\right) d\left(x_{n+1}, x_{\star}\right) \\
& =\left(k+\beta_{n}\right) d\left(x_{n}, x_{\star}\right)+d\left(f\left(x_{n}\right), T y_{n}\right)+\left(1-\beta_{n}\right) d\left(x_{n+1}, x_{\star}\right) .
\end{aligned}
$$

Thus, from (23) and (24), we have

$$
\left|d\left(f\left(x_{n}\right), T y_{n}\right)-d\left(f x_{\star}, x_{\star}\right)\right| \leq\left(k+\beta_{n}\right) d\left(x_{n}, x_{\star}\right)+\left(1-\beta_{n}\right) d\left(x_{n+1}, x_{\star}\right) .
$$

Using (25) and the fact that $x_{n} \rightarrow x \star$ as $n \rightarrow \infty$ in (22) we get that

$$
d^{2}\left(x_{\star}, q\right) \leq d^{2}\left(f x_{\star}, q\right)-d^{2}\left(f x_{\star}, x_{\star}\right) .
$$

That is,

$$
0 \leq \frac{1}{2}\left(d^{2}\left(x_{\star}, x_{\star}\right)+d^{2}\left(x_{\star}, q\right)\right) \leq d^{2}\left(f x_{\star}, q\right)-d^{2}\left(f x_{\star}, x_{\star}\right)=\left\langle\overrightarrow{x_{\star} f x_{\star}}, \overrightarrow{q x_{\star}}\right\rangle .
$$

Hence, from Lemma 1.0.8 $\chi_{\star}=P_{F(T)} f\left(\chi_{\star}\right)$ and this completes the proof.

Theorem 2.0.2. Let $C$ be a nonempty closed convex subset of a complete $C A T(0)$ space $E, T: C \rightarrow K(C) a$ multivalued nonexpansive mapping satisfying the endpoint condition and $f: C \rightarrow C$ a contraction with $k \in$ $(0,1)$. If sequences $\left\{\alpha_{n}\right\}$ and $\left\{\beta_{n}\right\} \in(0,1)$ satisfy
A1 $\lim _{n \rightarrow \infty} \alpha_{n}=0$,
A2 $\sum_{n=1}^{\infty} \alpha_{n}=\infty$,
A3 $\sum_{n=1}^{\infty}\left|\alpha_{n+1}-\alpha_{n}\right|=\infty$,
A4 $0<\epsilon \leq \beta_{n} \leq \beta_{n+1}<1$ for all $n \geq 0$

then the sequence generated by (3) converges strongly to a fixed point $x \star$ where $x_{\star} \in P_{F(T)} f\left(x_{\star}\right)$ which is equivalent to the following variational inequality

$$
\left\langle\overrightarrow{\chi_{\star} f\left(x_{\star}\right)}, \overrightarrow{x \chi_{\star}}\right\rangle \geq 0 \text { for all } x \in F(T) .
$$

Proof. The proof is similar to Theorem (2.0.1).

If $T$ is a nonexpansive single-valued operator, then from Theorem (2.0.1), we can obtain the following result in [3].

Theorem 2.0.3. Let $C$ be a nonempty closed convex subset of a complete $C A T(0)$ space $E, T: C \rightarrow C$ a nonexpansive mapping and $f: C \rightarrow C$ a contraction with $k \in(0,1)$. If sequences $\left\{\alpha_{n}\right\}$ and $\left\{\beta_{n}\right\} \in(0,1)$ satisfy
A1 $\lim _{n \rightarrow \infty} \alpha_{n}=0$,
A2 $\sum_{n=1}^{\infty} \alpha_{n}=\infty$,
A3 $\sum_{n=1}^{\infty}\left|\alpha_{n+1}-\alpha_{n}\right|=\infty$,
A4 $0<\epsilon \leq \beta_{n} \leq \beta_{n+1}<1$ for all $n \geq 0$

then the sequence generated by

$$
\begin{aligned}
x_{n+1} & =\alpha_{n} f\left(x_{n}\right) \oplus\left(1-\alpha_{n}\right) y_{n} ; \\
y_{n} & =\beta_{n} x_{n} \oplus\left(1-\beta_{n}\right) x_{n+1}
\end{aligned}
$$

converges strongly to a fixed point $x \star$ which is equivalent to the following variational inequality

$$
\left\langle\overrightarrow{x_{\star} f\left(\chi_{\star}\right)}, \overrightarrow{x x_{\star}}\right\rangle \geq 0 \text { for all } x \in F(T) .
$$


If $f$ is an identity function then we obtain the following result.

Theorem 2.0.4. Let $C$ be a nonempty closed convex subset of a complete $C A T(0)$ space $E$ and $T: C \rightarrow K(C)$ a multivalued nonexpansive mapping satisfying the endpoint condition. If sequences $\left\{\alpha_{n}\right\}$ and $\left\{\beta_{n}\right\} \in(0,1)$ satisfy

A1 $\lim _{n \rightarrow \infty} \alpha_{n}=0$,

A2 $\sum_{n=1}^{\infty} \alpha_{n}=\infty$,

A3 $\sum_{n=1}^{\infty}\left|\alpha_{n+1}-\alpha_{n}\right|=\infty$,

A4 $0<\epsilon \leq \beta_{n} \leq \beta_{n+1}<1$ for all $n \geq 0$

then the for $u \in C$ the sequence generated by

$$
\begin{aligned}
x_{n+1} & =\alpha_{n} u \oplus\left(1-\alpha_{n}\right) y_{n} ; \quad u_{n} \in T y_{n} ; \\
y_{n} & =\beta_{n} x_{n} \oplus\left(1-\beta_{n}\right) z_{n+1} ; \quad z_{n+1} \in T x_{n+1},
\end{aligned}
$$

such that $d\left(z_{n}, z_{n+1}\right) \leq d\left(x_{n}, x_{n+1}\right), d\left(u_{n}, u_{n+1}\right) \leq d\left(y_{n}, y_{n+1}\right)$ and $d\left(x_{n}, z_{n+1}\right) \leq d\left(x_{n}, x_{n+1}\right)$, converges strongly to a unique nearest point $x \star$ of $u$ in $F(T)$ which is equivalent to the following variational inequality

$$
\langle\overrightarrow{x \star u}, \overrightarrow{x x}\rangle \geq 0 \text { for all } x \in F(T) .
$$

Now, we prove some convergence results of the implicit viscosity approximation method in complete $\mathbb{R}$-trees without the endpoint condition.

Definition 2.0.5. An $\mathbb{R}$-tree is a geodesic space $E$ such that:

1. there is a unique geodesic segment $[x, y]$ joining each pair of points $x, y \in E$;

2. if $[y, x] \cap[x, z]=\{x\}$, then $[y, x] \cup[x, z]=[y, z]$;

3. if $u, v, w \in E$, then $[u, v] \cap[u, w]=[u, z]$ for some $z \in E$.

Every $\mathbb{R}$-tree is a CAT(0) space which does not contain the Euclidean plane. Thus to avoid the endpoint condition, $\mathbb{R}$-trees are preferred. Although an $\mathbb{R}$-tree is not strong enough to make all nonexpansive mappings having the endpoint condition, but it is strong enough to make our theorems hold without this condition.

Let $C$ be closed convex subset of a complete $\mathbb{R}$-tree $(E, d)$. Let $T: C \rightarrow K(C)$ be a multivalued mapping. Then, there exists a single-valued mapping $t: C \rightarrow C$ such that $t x \in T x$ and

$$
d(t x, t y) \leq H(T x, T y) \quad \text { for all } x, y \in C,
$$

see [22] for details. In this case, we call $t$ a nonexpansive selection of $T$. A multivalued mapping $T: C \rightarrow K(C)$ is called a quasi-nonexpansive mapping if

$$
H(T x, p) \leq d(x, p) \text { for all } x \in C, p \in F(T) .
$$

Clearly, every multivalued nonexpanive mapping is quasi-nonexpansive. The following result is needed in our result.

Proposition 2.0.6. [23] Let $C$ be a closed convex subset of a complete $\mathbb{R}-$ tree $(E, d)$ and $T: C \rightarrow P(C)$ a quasinonexpansive mapping with nonempty closed bounded convex values. Then $F(T)$ is closed and convex.

Let $f: C \rightarrow C$ be a contraction and fix $x_{1} \in C$. Define $\left\{x_{n}\right\} \in C$ by

$$
\begin{aligned}
x_{n+1} & =\alpha_{n} f x_{n} \oplus\left(1-\alpha_{n}\right) t y_{n} ; \quad t y_{n} \in T y_{n} ; \\
y_{n} & =\beta_{n} x_{n} \oplus\left(1-\beta_{n}\right) t x_{n+1} ; \quad t x_{n+1} \in T x_{n+1},
\end{aligned}
$$

where $d\left(x_{n}, t x_{n+1}\right) \leq d\left(x_{n}, x_{n+1}\right)$

and

$$
\begin{aligned}
x_{n+1} & =\alpha_{n} f x_{n} \oplus\left(1-\alpha_{n}\right) t y_{n} ; \quad t y_{n} \in T y_{n} ; \\
y_{n} & =\beta_{n} x_{n} \oplus\left(1-\beta_{n}\right) x_{n+1}
\end{aligned}
$$


for all $n \in \mathbb{N}$.

Theorem 2.0.7. Let $C$ be a nonempty closed convex subset of a complete $\mathbb{R}$-tree $E, T: C \rightarrow K(C)$ a multivalued nonexpansive mapping and $f: C \rightarrow C$ a contraction with $k \in(0,1)$. If sequences $\left\{\alpha_{n}\right\}$ and $\left\{\beta_{n}\right\} \in(0,1)$ satisfy

A1 $\lim _{n \rightarrow \infty} \alpha_{n}=0$,

A2 $\sum_{n=1}^{\infty} \alpha_{n}=\infty$,

A3 $\sum_{n=1}^{\infty}\left|\alpha_{n+1}-\alpha_{n}\right|=\infty$,

A4 $0<\epsilon \leq \beta_{n} \leq \beta_{n+1}<1$ for all $n \geq 0$.

Then the sequence generated by (29) converges strongly to a fixed point $x \star$ of $T$, which solves the variational inequality

$$
\left\langle\overrightarrow{x_{\star} f\left(x_{\star}\right)}, \overrightarrow{x X_{\star}}\right\rangle \geq 0 \text { for all } x \in F(T) .
$$

That is, $\chi_{\star} \in P_{F(T)} f\left(x_{\star}\right)$.

Proof. By Theorem 4.2 of [22], $F(t)=F(T)$. The set is closed and convex by Proposition 2.0.6 and $t$ is nonexpansive. The conclusion follows from Theorem 2.0.1.

Theorem 2.0.8. Let $C$ be a nonempty closed convex subset of a complete $\mathbb{R}$-tree $E, T: C \rightarrow K(C)$ a multivalued nonexpansive mapping and $f: C \rightarrow C$ a contraction with $k \in(0,1)$. If sequences $\left\{\alpha_{n}\right\}$ and $\left\{\beta_{n}\right\} \in(0,1)$ satisfy

A1 $\lim _{n \rightarrow \infty} \alpha_{n}=0$,

A2 $\sum_{n=1}^{\infty} \alpha_{n}=\infty$,

A3 $\sum_{n=1}^{\infty}\left|\alpha_{n+1}-\alpha_{n}\right|=\infty$,

A4 $0<\epsilon \leq \beta_{n} \leq \beta_{n+1}<1$ for all $n \geq 0$.

Then the sequence generated by (30) converges strongly to a fixed point $x_{\star}$ of $T$, which solves the variational inequality

$$
\left\langle\overrightarrow{x_{\star} f\left(\chi_{\star}\right)}, \overrightarrow{x \chi_{\star}}\right\rangle \geq 0 \text { for all } x \in F(T) .
$$

That is, $\chi \star \in P_{F(T)} f\left(\chi_{\star}\right)$.

Proof. The proof is similar to Theorem 2.0.7.

\section{Remarks}

Results presented in this paper extend corresponding results in [3] to multivalued nonexpansive mappings in the framework of CAT(0) spaces. Using Cauchy Schwarz inequality [19] and other results from [10], [8] etc. we have shown the strong convergence of the new viscosity method for the implicit rule of a multivalued nonexpansive mapping in $\operatorname{CAT}(0)$ space. However, in iterative method the condition $d\left(x_{n}, z_{n+1}\right) \leq d\left(x_{n}, x_{n+1}\right)$ on is imposed. Can these results be obtained without this condition? Furthermore, can these results be obtained when $f$ is a multivalued contraction mapping or $f$ satisfies other generalized contraction conditions? These are the open questions worth studying in the future.

Acknowledgements All the authors are grateful to the referees for their critical remarks and valuable suggestions which helped to improve the presentation of the paper. They also thank the Basque Government for Grant IT1207-19.

\section{References}

[1] Moudafi A., Viscosity approximation methods for fixed-points problems, J. Math. Anal. Appl., 2008, 241, 46-55

[2] Xu H. K., Alghamdi M. A., Shahzad N., The implicit midpoint rule for nonexpansive mappings in Banach spaces, Fixed Point Theory, 2016, 17(2), 509-517 
[3] Ke Y., Ma C., The generalized viscosity implicit rules of nonexpansive mappings in Hilbert spaces, Fixed Point Theory Appl., 2015, 2015:190

[4] Abkar A., Eslamian M., Convergence theorems for a finite family of generalized nonexpansive multivalued mappings in CAT (0) spaces, Nonlinear Anal., 2012, 75(4), 1895-1903

[5] Uddin I., Nieto J. J., Ali J., One-step iteration scheme for multivalued nonexpansive mappings in CAT (0) spaces, Mediterr. J. Math., 2016, 13(3), 1211-1225

[6] Uddin I., Ali J., Nieto J. J., An iteration scheme for a family of multivalued mappings in CAT(0) spaces with an application to image recovery, Rev. R. Acad. Cienc. Exactas Fés. Nat. Ser. A Math. RACSAM, 2018, 112(2), 373-384

[7] Wu X. B., Zhao L. N., Viscosity approximation methods for multivalued nonexpansive mappings, Mediterr. J. Math., 2016, $13,2645-2657$

[8] Preechasilp P., Viscosity approximation methods for implicit midpoint rule of nonexpansive mappings in geodesic spaces, Bull. Malays. Math. Sci. Soc., 2018, 41(3), 1561-1579

[9] Panyanak B., Suantai S., Viscosity approximation methods for multivalued nonexpansive mappings in geodesic spaces, Fixed Point Theory Appl., 2015, 2015:114

[10] Wangkeeree R., Preechasilp P., Viscosity approximation methods for nonexpansive mappings in CAT(0) spaces, Fixed Point Theory Appl., 2013, 2013:160

[11] Xiong T. J., Lan H. Y., Strong convergence of new two-step viscosity iterative approximation methods for set-valued nonexpansive mappings in CAT(0) spaces, J. Funct. Spaces, 2018, Article ID 1280241

[12] Xiong T. J., Lan H. Y., New two-step viscosity approximation methods of fixed points for set-valued nonexpansive mappings associated with contraction mappings in CAT(0) spaces, J. Comput. Anal. Appl., 2019, 26(5), 899-909

[13] Burago D. et al., A Course in Metric Geometry, vol. 33, Am. Math. Soc., Providence, 2001

[14] Bridson M., Haefliger A., Metric Spaces of Non-Positive Curvature, Springer, Berlin, 1999

[15] Brown K. S., Buildings, Springer, New York, 1989

[16] Dhompongsa S., Kirk W. A., On $\Delta$-convergence theorems in CAT(0) spaces, Comput. Math Appl., 2008, 56, 2572-2579

[17] Kirk W. A., Geodesic geometry and fixed point theory II, In: International Conference on Fixed Point Theory and Applications, Yokohama Publ., 2004, 113-142

[18] Chaoha P., Phon-on A., A note on fixed point sets in CAT(0) spaces, J. Math. Anal. Appl., 2006, 320(2), 983-987

[19] Berg I.D., Nikolaev I.G., Quasi linearization and curvature of Alexandrov spaces, Geom. Dedic., 2008, 133, 195-218

[20] Dehghan H., Rooin J., A characterization of metric projection in CAT(0) spaces, In: Proceedings of International Conference on Functional Equation, Geometric Functions and Applications (ICFGA), Tabriz, Iran, 2012, 41-43

[21] Xu H. K., An iterative approach to quadratic optimization, J. Optim. Theory Appl., 2003, 116, 659-678

[22] Aksoy A. G., Khamsi M. A., A selection theorem in metric trees, Proc. Am. Math. Soc., 2006, 134, 2957-2966

[23] Markin J., Fixed points for generalized nonexpansive mappings in R-trees, Comput. Math. Appl., 2011, 62(12), 4614-4618 